\title{
Four-dimensional multi-site photolysis of caged neurotransmitters
}

\author{
Mary Ann Go ${ }^{1}$, Minh-Son To ${ }^{1,2}$, Christian Stricker ${ }^{1,3}$, Stephen Redman ${ }^{1}$, Hans-A. Bachor ${ }^{4}$, \\ Greg J. Stuart ${ }^{1}$ and Vincent R. Daria ${ }^{1 *}$ \\ 1 Eccles Institute of Neuroscience, John Curtin School of Medical Research, The Australian National University, Canberra, ACT, Australia \\ 2 Department of Human Physiology and Centre for Neuroscience, Flinders University, Adelaide, SA, Australia \\ ${ }^{3}$ Medical School, College of Medicine, Biology and Environment, The Australian National University, Canberra, ACT, Australia \\ ${ }^{4}$ Department of Quantum Science, Research School of Physics and Engineering, The Australian National University, Canberra, ACT, Australia
}

\section{Edited by:}

Michael Hausser, University College

London, UK

Reviewed by:

Stéphane Dieudonné, IBENS, France Jeff Magee, Howard Hugues

Medical Institute, USA

\section{*Correspondence:}

Vincent R. Daria, John Curtin School of Medical Research, The Australian National University, Bldg. 131 Garran Road, Canberra, ACT 0200,

Australia

e-mail: vincent.daria@anu.edu.au
Neurons receive thousands of synaptic inputs that are distributed in space and time. The systematic study of how neurons process these inputs requires a technique to stimulate multiple yet highly targeted points of interest along the neuron's dendritic tree. Three-dimensional multi-focal patterns produced via holographic projection combined with two-photon photolysis of caged compounds can provide for highly localized release of neurotransmitters within each diffraction-limited focus, and in this way emulate simultaneous synaptic inputs to the neuron. However, this technique so far cannot achieve time-dependent stimulation patterns due to fundamental limitations of the hologram-encoding device and other factors that affect the consistency of controlled synaptic stimulation. Here, we report an advanced technique that enables the design and application of arbitrary spatio-temporal photostimulation patterns that resemble physiological synaptic inputs. By combining holographic projection with a programmable high-speed light-switching array, we have overcome temporal limitations with holographic projection, allowing us to mimic distributed activation of synaptic inputs leading to action potential generation. Our experiments uniquely demonstrate multi-site two-photon glutamate uncaging in three dimensions with submillisecond temporal resolution. Implementing this approach opens up new prospects for studying neuronal synaptic integration in four dimensions.

Keywords: two-photon photolysis, holographic projection, two-photon microscopy, caged neurotransmitters, synaptic integration

\section{INTRODUCTION}

Photostimulation using caged neurotransmitters (Callaway and Katz, 1993; Denk, 1994) and light-activated ion channels (Nagel et al., 2003; Boyden et al., 2005) have revolutionized the capacity to study the brain. Information processing in neuronal networks is achieved via signaling between neurons at synapses. Thus, controlled and localized activation of synapses offers the possibility to systematically study how individual neurons in the brain process information. Two-photon (2P) photolysis of caged compounds allows the simulation of highly localized release of neurotransmitter, enabling targeted activation of receptors at single synapses. Through activation of multiple synapses, it is possible to study how neurons integrate synaptic inputs to generate action potentials (APs). However, to date it has not been possible to activate synapses arbitrarily along the three-dimensional dendritic arbor of a neuron using realistic spatio-temporal [i.e., four-dimensional (4D)] patterns of light that resemble physiological inputs.

Laser-scanning systems utilizing galvanometer (GM) mirrors (Gasparini and Magee, 2001; Matsuzaki et al., 2001; Branco et al., 2010) implemented in conventional two-photon laser-scanning microscopes provide quasi-simultaneous multi-site stimulation by scanning the laser beam at fast rates $(\sim 10 \mathrm{kHz}$; i.e., switching time of $\sim 100 \mu \mathrm{s})$. These scanning systems, however, are two-dimensional and are restricted to a single optical plane. A high-speed 3D scanning system has been described utilizing four acousto-optic modulators (AOMs) (Reddy et al., 2008). This system has been used to monitor neuronal activity via calcium imaging (Reddy et al., 2008; Katona et al., 2012). However, using four AOMs results in low optical throughput, and the dispersion through the AOM crystal significantly decreases $2 \mathrm{P}$ efficiency due to pulse broadening. Consequently, such a system has not been utilized for multi-site photolysis of caged neurotransmitters in $3 \mathrm{D}$.

Beam-shaping techniques using a spatial light modulator (SLM) can split a single laser beam into several beamlets and generate arbitrary illumination patterns for photostimulation. These techniques have been demonstrated for photostimulation with shaped illumination in single-photon (1P) (Lutz et al., 2008; Zahid et al., 2010) and 2P (Dal Maschio et al., 2010; Papagiakoumou et al., 2010), and for a multi-foci photostimulation pattern in 1P (Anselmi et al., 2011; Yang et al., 2011) and 2P (Nikolenko et al., 2008). 1P excitation exhibits poor localized excitation along the optical axis. While $2 \mathrm{P}$ photostimulation offers the required axial resolution, previous studies have only showed stimulation in a single plane. We recently demonstrated $2 \mathrm{P}$ photolysis of caged neurotransmitters using holographic 
projection of an arbitrary 3D multi-foci uncaging pattern (Go et al., 2012). While holographic projection allows simultaneous multi-site photostimulation in $3 \mathrm{D}$, the slow response of the SLM ( 10-30 ms) remains the limiting factor in achieving fast switching light patterns within physiologically relevant timescales $(\sim 1 \mathrm{~ms})$. Here, we overcome this limitation by temporally gating the 3D multi-foci uncaging pattern generated by the SLM using a high-speed spatial light switching array provided by a digital micro-mirror device (DMD). The programmable array of micro-mirrors can be used to independently control each beamlet in less than a millisecond $(\sim 0.7 \mathrm{~ms})$ allowing the multi-site pattern to be changed with submillisecond speed. Random spatial stimulation patterns in 3D are therefore possible within physiologically relevant timescales. We demonstrate the performance of such a system as a tool for studying neuronal integration by uncaging glutamate at multiple foci with submillisecond resolution and thereby stimulate dendritic spines on different dendritic branches.

\section{MATERIALS AND METHODS 2.1. MICROSCOPE DESIGN}

Our time-gated holographic microscope system employs an SLM, encoded with a phase hologram to generate a multi-focal excitation pattern from the incident laser beam, and a DMD, which acts as a spatial light switch allowing independent switching of the individual foci. The resulting spatio-temporal excitation pattern is used for glutamate uncaging. We also use GM scanning mirrors to image the 3D morphology of the neuron. A schematic of the system is described in Figure 1A. Two laser beams for imaging $(800 \mathrm{~nm})$ and uncaging $(720 \mathrm{~nm})$, respectively, are combined ahead of the objective lens via a polarizing beam splitter following re-orientation of the polarization of each laser using half-wave plates. GM scanning mirrors scan the linearly polarized imaging beam from a near infrared (NIR) Ti:S laser (Coherent Inc. MIRA 900) for 2P fluorescence imaging and a dichroic mirror reflects the beam to the objective. We obtain a $2 \mathrm{P}$ image by collecting the green fluorescence from the sample directed to a second dichroic mirror, which reflects wavelengths below $650 \mathrm{~nm}$ into a photomultiplier tube. The sample may also be viewed via an upright differential interference contrast (DIC) microscope (Olympus BX50WI). In DIC imaging mode, the dichroic mirror above the objective lens allows infrared light $(>810 \mathrm{~nm}$ wavelength) to pass through and focus onto a charge-coupled device (CCD) camera (Dage-MTI IR-1000EX). The uncaging laser beam from a Ti:S laser (Coherent Inc. Chameleon) is expanded by a telescope to illuminate the $16 \times 12 \mathrm{~mm}^{2}$ area of a programmable phase-only SLM (Hamamatsu X10468-02), where the phase-only hologram is encoded. The hologram is computed using custom software based on the standard prism-lens superposition algorithm (Liesener et al., 2000; Curtis et al., 2002). The DMD (DLP3000, Texas Instruments) is located at the Fourier plane with respect to the SLM and is interfaced to the microscope using $4 f$ relay lenses, which position the DMD at the conjugate image plane with respect to the sample region. The DMD is used to

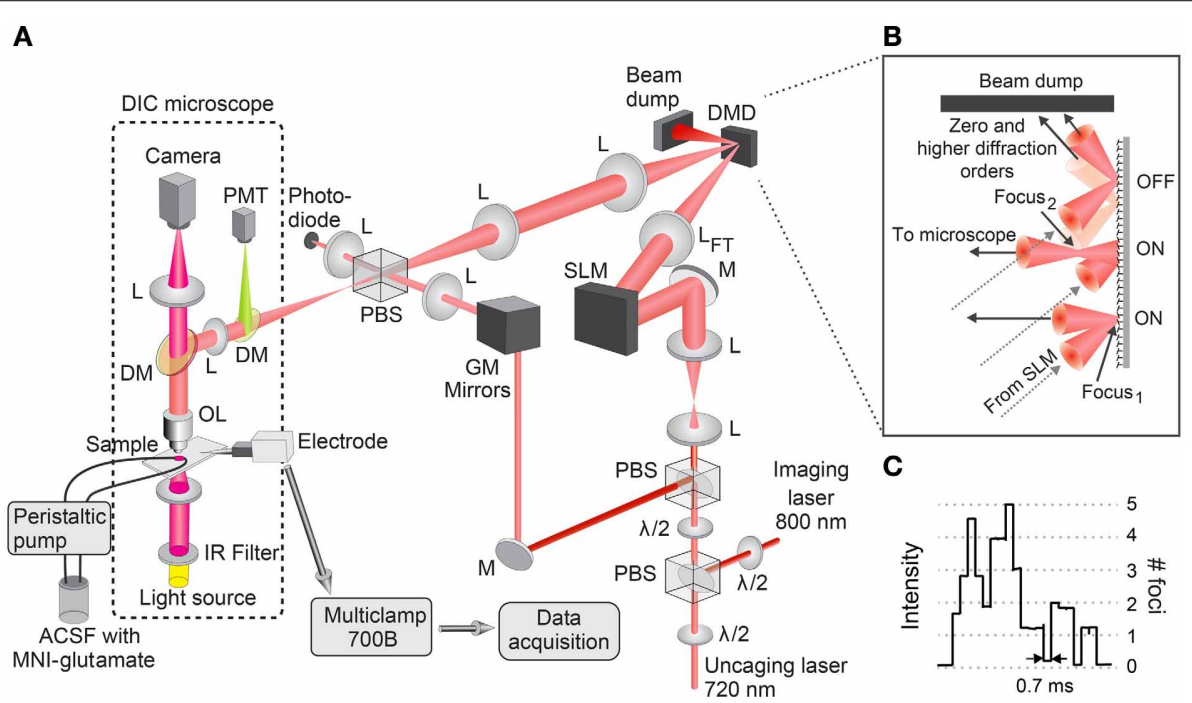

FIGURE 1 | Optical design of 4D holographic microscope. (A) Light from a laser tuned to $800 \mathrm{~nm}$ is projected through two polarizing beam splitters (PBS) onto two galvanometer (GM) scanning mirrors that scan the excitation beam across the sample for imaging. Fluorescence from the sample is collected by a photomultiplier tube (PMT). Light from another laser tuned to $720 \mathrm{~nm}$ is expanded, projected onto a spatial light modulator (SLM), focused onto a digital micro-mirror device (DMD) and imaged onto the sample. The SLM is encoded with a phase hologram that generates a multi-focal excitation pattern from the incident laser beam. The DMD acts as a spatial light switch allowing independent switching of the individual foci. The resulting spatio-temporal excitation pattern at the sample plane is used for uncaging glutamate. $\lambda / 2$, half-wave plate; $D M$, dicroic mirror; $M$, mirror; $L$, lens; $L_{F T}$, Fourier transform lens; OL, objective lens; ACSF, artificial cerebro-spinal fluid. (B) The DMD switches the excitation focus ON and OFF by changing the tilt of the subset of programmable micro-mirrors in the corresponding focus (Focus ${ }_{1}$ if confocal with the objective lens). A larger subset of micro-mirrors is needed when the beam focus (Focus 2 ) is off the DMD plane. In the ON state, the laser beam is directed to the objective lens; in the OFF state, to a beam dump. Zero and higher diffraction orders are also directed to the beam dump. (C) The number of foci turned $\mathrm{ON}$ is indicated by the laser intensity incident on the sample measured using the photodiode located in the conjugate Fourier plane. Minimum switching time with the DMD is $0.7 \mathrm{~ms}$. 
individually switch each beamlet of the excitation pattern $\mathrm{ON}$ or OFF. In the ON state, the micro-mirrors direct the laser light to the sample (see Figure 1B). In the OFF state, the laser light is directed to a beam dump. The relay lenses are chosen to ensure that the back aperture of the objective lens is filled and that the area of the DMD encompasses the field of view of the objective lens $\left(200 \mu \mathrm{m}^{2}\right.$ for a $1.0 \mathrm{NA} 40 \times$ objective $)$.

The dimension of one micro-mirror in the DMD is smaller than the diffraction-limited focal spot of the Fourier transform (FT) lens, with an effective numerical aperture (NA) of $\sim 0.1$. Hence, it takes a small set of micro-mirrors to switch a single beamlet of the multi-focal pattern $\mathrm{ON}$ and direct it to the sample for uncaging (see Figure 1B). When the holographic focus generated by the SLM is either above or below the plane of the DMD, a larger subset of micro-mirrors is needed. The size of the subset of micro-mirrors is calculated based on the conical angle of the focus with respect to the NA of the FT lens and the operating wavelength $(720 \mathrm{~nm})$. Figure $1 \mathrm{C}$ shows the relative intensity in the sample plane as measured by a photo-diode located at the conjugate Fourier plane (see Figure 1A) for different numbers of focal spots turned ON by the DMD. The intensity is more or less quantized and gives information on the number of foci active for uncaging. The DMD receives 24 bit RGB data at $60 \mathrm{~Hz}$ through its electronic video interface, with each color channel (RGB) having 8-bit depth. We operated the DMD in 1-bit monochrome mode. In this mode, a single image frame consists of 24 1-bit planes which encode the micro-mirror state at consecutive time epochs. Since each plane is weighted equally, this allows temporal sequences to be read out at $1440 \mathrm{~Hz}$. By driving the DMD this way, the minimum switching time for a full ON-OFF cycle is $0.7 \mathrm{~ms}$ (see Figure 1C).

The setup is also equipped with a micromanipulator (Sutter Instruments), peristaltic pump (Gilson Minipuls 3) and amplifier (MultiClamp 700B, Molecular Devices) for electrophysiology. We use custom software developed in Labview (National Instruments) to control the acquisition of 3D 2P images, the calculation of the appropriate hologram for projection of photostimulation sites, and the laser intensity via a polarizing beam splitter and a half-wave plate on a motorized rotation mount.

\subsection{TISSUE PREPARATION AND ELECTROPHYSIOLOGY}

Three hundred micrometer thick slices of rat somatosensory cortex and hippocampus (P22-35) were prepared with a vibratome (Leica VT1200S). The slices were cut in ice-cold oxygenated artificial cerebrospinal fluid (ACSF) that contained (in $\mathrm{mM}): 1.25 \mathrm{NaH} 2 \mathrm{PO} 4,1.0 \mathrm{MgCl} 2,125.0 \mathrm{NaCl}, 2.5 \mathrm{KCl}, 2.0 \mathrm{CaCl}$, $25.0 \mathrm{NaHCO} 3$, and 10.0 glucose. Slices were incubated in oxygenated $\mathrm{ACSF}$ at $34^{\circ} \mathrm{C}$ for $30 \mathrm{~min}$ and kept at room temperature before being transferred to the recording chamber. Animal experiments were performed with the protocol approved by the Animal Ethics Committee of the Australian National University.

We filled neurons through the recording patch pipette $(R=$ 3.5-4.5 M $\Omega$ ) with an intracellular solution containing (in $\mathrm{mM}$ ): $115 \mathrm{~K}$-gluconate, $20 \mathrm{KCl}, 10 \mathrm{HEPES}, 10$ phospho-kreatine, 4 ATP$\mathrm{Mg}, 0.3 \mathrm{GTP}, 5.4$ biocytin, and 0.1 Alexa-488 (Invitrogen). Whole-cell current clamp recordings of layer II/III and V pyramidal cells were obtained with a MultiClamp 700B. Current was injected when necessary to maintain a resting membrane potential of $-70 \mathrm{mV}$. For the experiments in Figures 3D-F, the resting membrane potential was depolarized to $-55 \mathrm{mV}$ to allow for APs to be more easily generated. Data analysis was done with AxoGraph X and Matlab. We calculated peak currents and voltages by averaging 5-12 trials.

\subsection{TWO-PHOTON IMAGING}

Neurons filled with $0.1 \mathrm{mM}$ Alexa-488 were imaged at $800 \mathrm{~nm}$ with $12-22 \mathrm{~mW}$ laser power. The dye was allowed to diffuse into the neuron for 20-30 min before imaging. Image stacks of $800 \times$ 800 pixels in a single plane were generated by imaging individual planes in $1 \mu \mathrm{m}$ increments along the $z$-axis. ImageJ (National Institute of Health) was used for $3 \mathrm{D}$ visualization.

\subsection{TWO-PHOTON GLUTAMATE UNCAGING}

We determined the potential sites on the dendritic tree for photostimulation from the 3D image map of the fluorescently labeled neuron. The appropriate phase hologram for the desired multifocal pattern around the neuron was then calculated and encoded onto the SLM. The DMD was used to switch individual sites ON or OFF.

MNI-caged glutamate (Tocris Bioscience) was bath-applied $(3 \mathrm{mM})$ and uncaged at $720 \mathrm{~nm}$ in the presence of $0.1 \mathrm{mM}$ cyclothiazide using 9-30 $\mathrm{mW}$ power per uncaging spot. A closed recirculating system using a peristaltic pump was used to minimize the ACSF volume. An automated drift correction algorithm was run before every uncaging event to ensure that the photostimulation sites remained optimized.

\section{RESULTS}

\subsection{TIME-GATED HOLOGRAPHIC SYSTEM}

Our microscope design builds on an earlier reported two-photon holographic microscope system capable of simultaneous multisite photostimulation in 3D (Go et al., 2012). The incorporation of a DMD allows for full-cycle (OFF-ON-OFF) switching of photostimulation patterns within $0.7 \mathrm{~ms}$. This is a rate that is not possible with just the SLM alone, which has a response time of 10-30 ms. Although high-speed shutter systems can operate on the same submillisecond timescale, they do not allow independent switching of multiple foci. The addition of the DMD to our set-up also "cleans up" the 3D multi-site photostimulation pattern produced by the SLM. The DMD offers a convenient way to eliminate the spurious light patterns that normally result from holographic projection using a phase-only SLM with finite space-bandwidth product (Palima and Daria, 2006, 2007; Go et al., 2011). To visualize the different foci, we placed a reflective surface (e.g., glass slide) in the sample plane and imaged the reflected light leaked through the dichroic mirror directly above the objective lens (see Figure 1A). Figure 2A shows the photostimulation pattern produced by the SLM projected onto the sample with all DMD pixels in the ON position. Figure $2 \mathbf{B}$ shows the same photostimulation pattern with only the intended nine foci switched ON. Note that the undiffracted (i.e., zero-order; see image center in Figure 2A) beam from the SLM is missing in the latter, as are other higher diffraction orders and mirror projections of the first orders. The DMD therefore provides an easy 

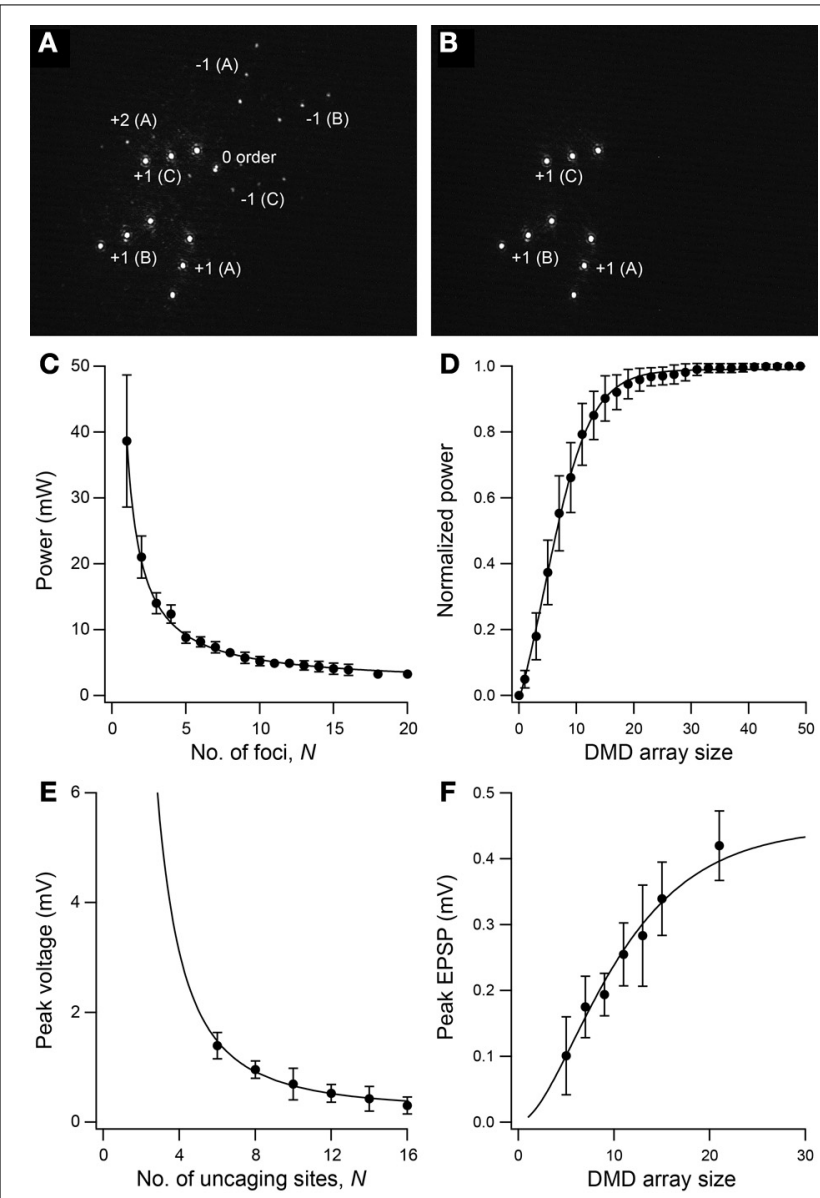

FIGURE 2 | SLM-generated 3D photostimulation pattern with DMD gating. (A) Nine-foci light pattern produced by the SLM with all DMD pixels turned ON showing zero order (0 order), second order $[+2(A)]$ and mirror projections $[-1(A-C)]$ of the first diffraction order $[+1(A-C)]$. (B) Same photostimulation pattern as in (A) but with only the nine desired foci gated by the DMD. All other light is eliminated by being directed to the beam dump. (C) Power on one ON focus as a function of number of foci, $N$ ( $n=10$ different spot configurations). For $N=2-20$, the ON focus was kept at a fixed position. Solid line is $1 / N$ fit. (D) Normalized power as a function of DMD array size ( $n=10$ focal spot positions). Solid line is a cumulative Gaussian fit. (E,F) Peak of glutamate-evoked voltage from one ON uncaging site as a function of $(\mathbf{E})$ number of uncaging sites and $(\mathbf{F})$ DMD array size ( $n=8-10$ trials). Solid lines are proportional to squares of curve fits in $C$ and $D$, respectively.

way of eliminating the zero-order beam, thus, eliminating the risk of unintended uncaging, and gives independent control for switching each stimulation site.

Figure 2C shows how the power on one uncaging focus depends on the number of focal spots, $N$. The relationship is an inverse proportionality as reported earlier (Daria et al., 2009). The error bar for $N=1$ shows the variability in power among different non-central positions of the holographic spot which results from the spatial variation in the diffraction efficiency of the SLM. For $N=2$ to 20 , the $\mathrm{ON}$ focus was kept at a fixed position as the spot configuration for a fixed number of foci was varied. Note that the variability in power within a single position is much less than the spatial variability. The laser beam has a Gaussian intensity profile. Figure 2D shows the normalized power as a cumulative Gaussian function of DMD array size, which defines the length of the square array of micro-mirrors for gating the uncaging focus, averaged over different positions of the holographic spot. On average, $96 \%$ of the maximum intensity at each spot was recovered using a DMD array size of 21 , whereas $90 \%$ was recovered with an array size of 15 . Based on this information, for most of the experiments in this paper we used an array size of 15 . Figures 2 E,F show how the glutamate uncaging-evoked response varies with number of uncaging sites, $N$, and DMD array size, respectively. The quadratic intensity dependence of $2 \mathrm{P}$ absorption is reflected here with the peak voltage seen as a function of $1 / N^{2}$ and a squared cumulative Gaussian function of DMD array size. As the total laser power was kept constant for the experiment in Figure 2E, data points for $N<6$ were not taken to preclude damaging the DMD with high laser power. Laser pulse duration for Figures 2E,F is $2 \mathrm{~ms}$.

Figure $3 \mathrm{~A}$ shows 11 foci positioned in a circular arrangement with $z$-axis positions ranging from $10 \mu \mathrm{m}$ above (negative) to $10 \mu \mathrm{m}$ below (positive) the nominal focus of the objective lens $(0 \mu \mathrm{m})$. The point in the focal plane is in focus (i.e., sharpest) but the rest are out of focus with the points in the farthest away planes $( \pm 10 \mu \mathrm{m})$ the most out of focus. Figure 3B shows the same light pattern but with the objective lens focused in the plane $z=+10 \mu \mathrm{m}$. Figure 3C shows the DMD array sizes for gating the different foci in the light pattern in Figure 3A. By scaling the micro-miror array size for gating the relevant site with distance from the nominal focal plane of the objective lens, we accommodate the increasing size of the out-of-focus projection of the beamlets and allow collection of light for points projected above and below the focal plane. The DMD array size puts a restriction on the minimum separation between two uncaging sites for independent gating. Figure 3D plots the minimum distance between two uncaging sites as a function of axial distance for an array size of 15 for the case where one spot is fixed while the other spot is moved away axially and for when both spots move together in the axial direction. The deviation of data points from a straight line is due to the DMD having a finite number of discrete pixels. The minimum separation for two uncaging sites in the same plane is $6.5 \mu \mathrm{m}$. We refrained from positioning uncaging sites within 10 $\mu \mathrm{m}$ of the center to avoid switching $\mathrm{ON}$ the zero-order beam and unnecessarily illuminating the sample. Technically, however, this minimum separation is a soft limit as independent uncaging at two sites, defined by the spatial resolution of glutamate uncaging, has a much narrower profile. Figures $3 E, F$ show representative lateral and axial resolution profiles from uncaging-evoked voltage responses to $700 \mu$ s laser pulse duration. The responses were measured in current clamp as the uncaging site was moved orthogonally from the spine while the objective was kept fixed. The lateral profile has a full width at half maximum (FWHM) of $0.8 \pm 0.1 \mu \mathrm{m}$. The axial profile was $1.3 \pm 0.1 \mu \mathrm{m}$. The solid lines correspond to Gaussian curve fits. The final resolution depend on laser power, laser pulse duration and local MNI-glutamate concentration. For example, we earlier reported uncaging resolution with FWHM of $2.4 \pm 0.2 \mu \mathrm{m}$ and $3.7 \pm 0.3 \mu \mathrm{m}$ for the lateral 

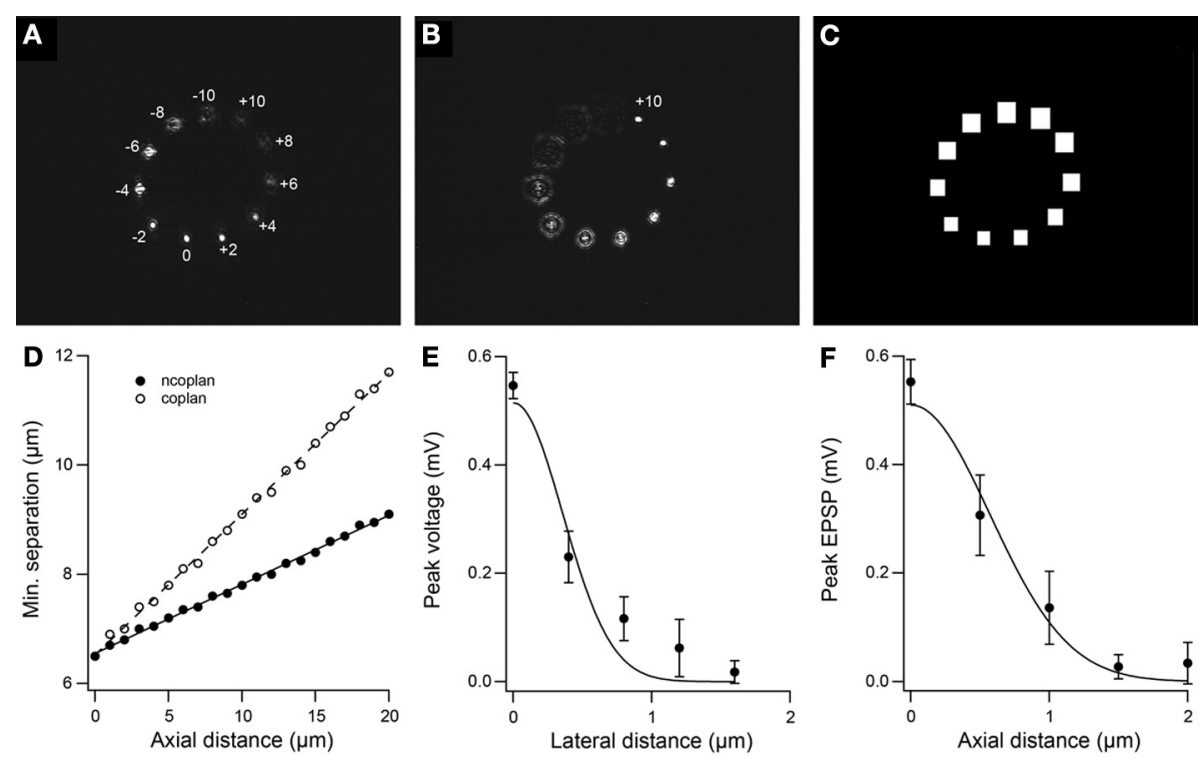

FIGURE 3 | Spatial resolution of independent gating and uncaging. (A) 3D photostimulation pattern with objective lens focused in the reference plane $z=0 \mu \mathrm{m}$ and the other 10 sites holographically projected either above (negative) or below (positive) the reference plane. (B) Same photostimulation pattern as in $C$ but with objective lens focused in the plane $z=+10 \mu \mathrm{m}$. (C) Sizes of micro-mirror arrays for switching foci shown in $A$. The array size scales with distance from the objective focal plane allowing collection of out-of-focus light. (D) Minimum separation for independent gating of two uncaging sites as a function of axial distance for the case when one spot is fixed while the other spot is moved away axially (ncoplan) and for when both spots move together in the axial direction (coplan). DMD array size is 15. Solid line is linear fit. (E) Peak voltage with varying lateral distance orthogonal to a spine. $\mathrm{FWHM}=0.8 \pm 0.1 \mu \mathrm{m}$. (F) Peak voltage as a function of axial distance above the spine. FWHM $=1.3 \pm 0.1 \mu \mathrm{m}$. For $E$ and $F$, solid lines are Gaussian fits. and axial profiles, respectively, using $2 \mathrm{~ms}$ laser pulse duration (Go et al., 2012).

\subsection{SPATIO-TEMPORAL PATTERNED STIMULI}

The system offers access to the 3D dendritic arbor of the neuron for activating synaptic receptors. This enables the study of how neurons integrate multiple synaptic inputs on their dendritic trees. We illustrate such an experiment in Figure 4. A 2P fluorescence image of a layer $\mathrm{V}$ pyramidal neuron is shown in Figure 4A with the recording pipette at the soma and some basal dendrites either viewed from the top $(x y)$ or side $(y z)$. Four uncaging sites are chosen with two each projected onto two basal dendrites located $5 \mu \mathrm{m}$ apart in the vertical direction. A static hologram for all four sites is generated with the SLM, and the DMD is used to switch $\mathrm{ON}$ one focus at a time. Figure 4B shows glutamateevoked excitatory postsynaptic potentials (EPSPs) generated by individually uncaging glutamate at each site for a duration of $3 \mathrm{~ms}$. Laser power was set to generate EPSPs consistent with single synaptic inputs ( $<0.5 \mathrm{mV}$ ) (Nevian et al., 2007; Fino et al., 2009). Figure 4C summarizes EPSP summation for all the possible combinations of the uncaging sites. Linearity is expressed as the ratio of the peak amplitude of the composite EPSP generated when all sites are simultaneously stimulated and the arithmetic sum of individual EPSPs. The blue bar corresponds to within-branch and the red to between-branch summation, respectively. No statistically significant difference is observed, indicating that the summation is largely linear (two inputs in one basal, $n=2$; two inputs on two basal dendrites, $n=4$; three inputs, $n=4$; four inputs, $n=1$; pooled data, $n=11$ combinations). This observed linear within-branch summation is consistent with the observation by Polsky et al. (2004) who showed that two weak EPSPs on the same layer $\mathrm{V}$ basal dendrite $20 \mu \mathrm{m}$ apart summate linearly.

The DMD, by enabling independent switching of individual photostimulation sites, makes it possible to activate different combinations of the sites without having to change the phase hologram; i.e., the hologram remains static and sites are switched ON/OFF by the DMD. Moreover, as different stimulus combinations need to be compared, it is critical that the power at each uncaging site is kept constant. The DMD allows this and eliminates the necessity to adjust the total power for distribution among the uncaging sites when using different numbers of stimulus sites in this experiment. In addition, because the hologram is kept static and the power at each site constant, the power variability due to the spatially varying diffraction efficiency of the SLM is not an issue.

Next, we explored if fast multi-site photostimulation patterns could elicit APs. Figure 4D shows five marked uncaging sites on three apical oblique dendrites of a layer II/III pyramidal neuron. The DMD is used to randomly switch uncaging sites ON or OFF for $0.7-4 \mathrm{~ms}$ at a time for a total period of $4 \mathrm{~s}$. Figure $4 \mathrm{E}$ shows the relative intensity at the sample, indicating the number of stimulus sites at which neurotransmitter is released due to uncaging, together with the membrane potential at the soma for two trials of identical time-varying uncaging patterns. Several peaks of the APs during the two trials align in time indicating that the APs are not randomly evoked, but are triggered by specific uncaging patterns. Figure $4 \mathrm{E}^{\prime}$ shows a magnified view of sections of the membrane potential trajectory and laser intensity $60 \mathrm{~ms}$ before to $20 \mathrm{~ms}$ after 


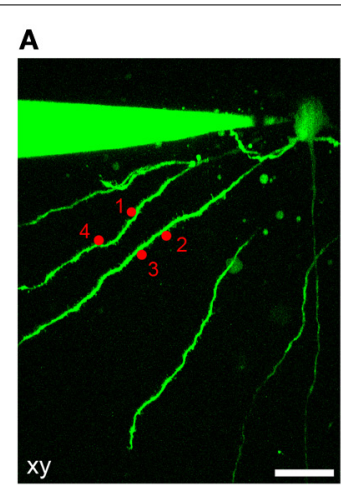

D

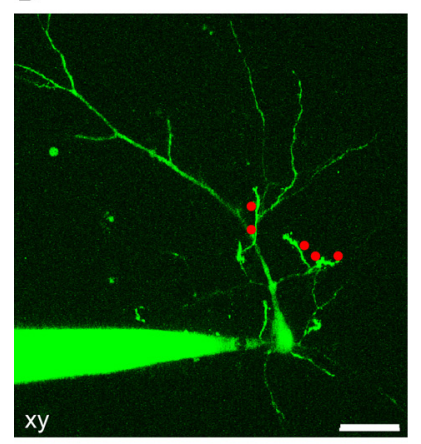

$\mathbf{F}$

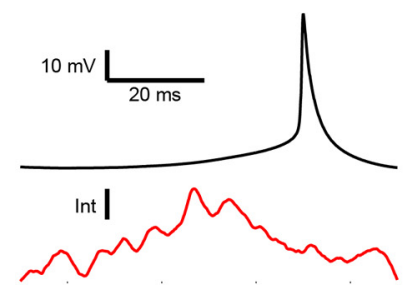

B

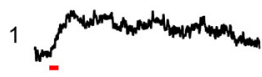

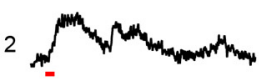

(3)

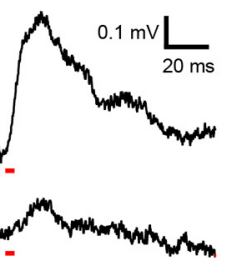

C

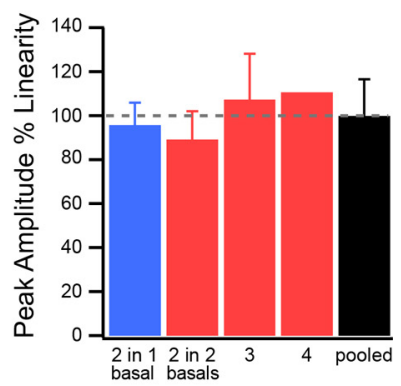

No. of inputs

E
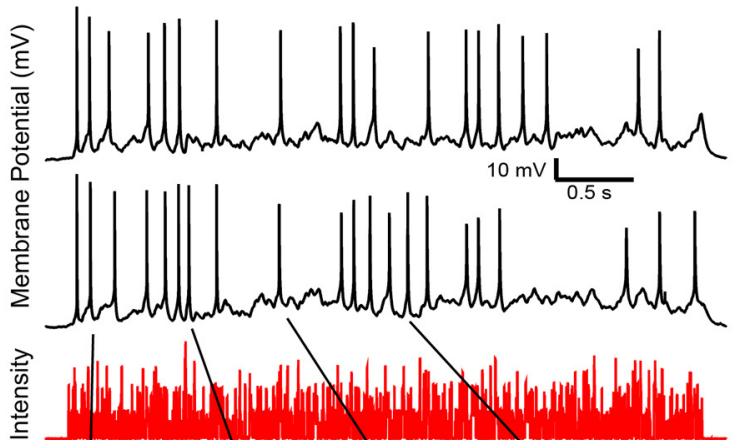

E' $^{\prime}$

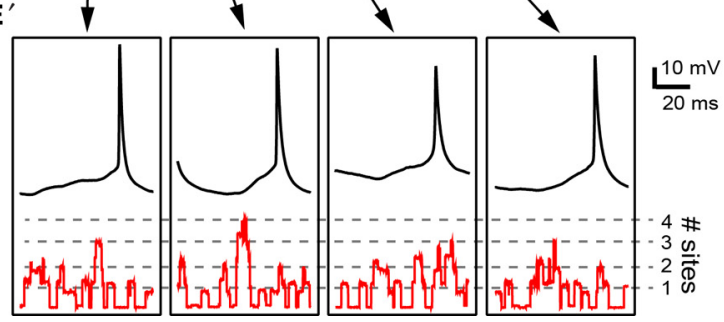

FIGURE 4 | Synaptic integration experiments. (A) Layer V pyramidal cell with uncaging sites in two planes $5 \mu \mathrm{m}$ apart (1 and 2 are in one plane, 3 and 4 in another) as viewed in the $x y$ and $y z$ planes. Scale bar $25 \mu \mathrm{m}$. (B) Individual uncaging-evoked EPSPs obtained by turning ON stimulation sites in a static hologram one at a time with the DMD. Stimulus duration $(3 \mathrm{~ms})$ is indicated by red bars at the start of the EPSP. (C) Summary of results for different combinations of uncaging sites. Linearity is expressed as the ratio of the peak amplitudes of the measured compound EPSP and the arithmetic sum of individual EPSPs. Dashed line denotes linear summation (100\%). Blue bar corresponds to within-branch summation; red, between-branch summation. Data are shown as mean \pm s.e.m. for all combinations. (D) Layer II/III pyramidal cell with five uncaging sites situated along three apical oblique branches and all in a single plane. Scale bar $25 \mu \mathrm{m}$. (E) Representative membrane potential time courses generated by two repeats of the same train of random stimulus for uncaging glutamate at the sites in $D$. In the stimulus, the spatial selections and their time intervals are both random. The resting membrane potential at the soma was set to $-55 \mathrm{mV}$. Several action potentials (APs) align in time triggered by the same uncaging patterns. Laser intensity in the sample plane is indicative of the number of simultaneously active stimulus sites. (E') Magnified view showing membrane potential (black) and laser intensity (red) for a time window from $60 \mathrm{~ms}$ before to $20 \mathrm{~ms}$ after the peak of the AP. (F) Mean membrane potential and laser intensity ( $n=53$ APs) from $60 \mathrm{~ms}$ preceding peak of AP to $20 \mathrm{~ms}$ after. AP is driven by a significant increase in laser intensity shortly $(\sim 23 \mathrm{~ms})$ before its peak. the peak for four of the APs generated by this uncaging pattern. Integration of several inputs is required prior to AP generation. Figure 4F shows the average membrane potential and laser intensity ( $n=53$ APs) from $60 \mathrm{~ms}$ preceding peak of the AP to $20 \mathrm{~ms}$ after. On average, an AP is driven by a significant increase in laser intensity $\sim 23 \mathrm{~ms}$ before its peak.

\section{DISCUSSION}

\subsection{APPLICATIONS OF A TIME-GATED HOLOGRAPHIC SYSTEM IN NEUROSCIENCE}

We have shown that holographic projection with high-speed temporal gating via a DMD overcomes the 2D limitation of
GM-based laser scanning systems and the poor temporal resolution of SLM-based holographic projection systems for $2 \mathrm{P}$ uncaging of neurotransmitters. The DMD allows independent control of each focus in the SLM-generated photostimulation pattern and offers a convenient way to eliminate the zero and higher diffraction orders. This method offers unprecedented flexibility in the design of spatio-temporal (or 4D) light patterns for highly localized release of neurotransmitters. It opens up a fresh possibility to study synaptic integration in 3D with high temporal resolution. Since holographic projection allows easy positioning of photostimulation sites along the dendritic arbor of neurons, this method allows integration between branches to be directly 
investigated. This is crucial since in pyramidal neurons, which are abundant in several regions of the central nervous system in mammals (Spruston, 2008), apical oblique and basal dendrites collectively receive the vast majority of excitatory synapses (Larkman, 1991; Megias et al., 2001; Wang et al., 2007). This method could also be applied to the spatio-temporal control of neuronal activity which allows probing of neural coding, i.e., what kinds of spatio-temporal input patterns a neuron responds to. In addition, uncaging may be extended to neuronal populations. By generating a multi-cell holographic stimulation pattern and using the DMD to sequentially evoke suprathreshold $2 \mathrm{P}$ activation of neurons in a population, connections to a patched neuron may be determined. This facilitates a fast method for finding connected pairs, for instance. This multiplexing technique may also be used for population calcium imaging in the study of network activity (Ducros et al., 2013) and the interaction between different subnetworks. Another potential field of application for these 4D light patterns is optogenetics. By using sculptured light to activate specific subnetworks of genetically labeled neuronal populations, an even higher degree of selectivity can be achieved than is possible with traditional optogenetic approaches alone (Liu et al., 2012; Lovett-Barron et al., 2012).

\subsection{RELATIONSHIP WITH OTHER WORK}

The basic component of holographic projection is a phase-only liquid crystal (LC) SLM encoded with a computer-generated phase hologram. The response time of the LC is dependent on its phase retardation rate, which relates to the operating wavelength of the SLM (Wu, 1986). This response time is typically $\sim 10 \mathrm{~ms}$, but addressing via computer video output further limits the temporal resolution to $\sim 30 \mathrm{~ms}$. There have been several attempts to increase the response time of SLMs (Dayton et al., 2001; Kirby and Love, 2004), but no technique using spatially addressable SLMs has achieved multi-level phase-only modulation to perform a full OFF-ON-OFF cycle of the hologram at submillisecond timescales. Thalhammer et al. (2013) recently demonstrated highspeed hologram transition using a device with an extended phase map of up to $4 \pi$ and choosing the minimum route in phase shift for each pixel in between transitions. This technique allows for one-way transitions of 1-3 ms. An iterative hologram optimization routine is necessary to improve the diffraction efficiency with the reduced phase representation of the hologram. While this technique can potentially be applied to $4 \mathrm{D}$ photostimulation, changing the hologram introduces spurious light patterns during hologram transitions, which could result in unwanted uncaging. While a high-speed shutter or an acousto-optic deflector can be used to turn OFF the light during transitions, the projection of zero and higher diffraction orders can still pose a potential issue when the hologram is ON especially when the iterative optimization routine does not produce an efficient hologram for a specific stimulation pattern.

Holographic projection with binary phase modulations ( 0 and $\pi$ ) has been demonstrated recently for the study of neuronal circuits (Reutsky-Gefen et al., 2013). SLMs employing ferroelectric LCs have fast response times $(\sim 0.5 \mathrm{~ms})$. However, binary holograms produce mirror projections of equal light intensity in addition to the zero order. This reduces the power available for stimulation (after blocking zero order and mirror projection) to significantly less than $40 \%$. While such technique can well be applied for $1 \mathrm{P}$ excitation, either for optogenetics or uncaging, its low optical throughput can be an issue when applying the technique to $2 \mathrm{P}$ uncaging in $4 \mathrm{D}$. Moreover, even for a fixed number of foci, global changes in the binary hologram impact the intensity level of each focus and could introduce variable $2 \mathrm{P}$ uncaging responses. Changing the number of foci necessitates dynamic control of the laser intensity affecting both $1 \mathrm{P}$ and $2 \mathrm{P}$ uncaging modes.

Our technique uniquely distinguishes itself from existing techniques in that it uses a conventional phase-only SLM with slow refresh rate combined with a DMD, which enables independent gating of the holographically projected multiple foci at high speeds. Here, the SLM first modulates the phase of the incoming laser beam to generate the desired multi-focal spatial profile before the DMD allows each focus to be individually turned ON or OFF. Thus, there is minimal loss of laser intensity compared to the method of using DMDs to remove unwanted light from a wide-field illumination to shape the excitation light (Bednarkiewicz et al., 2008; Zhu et al., 2012).

\subsection{ChALLENGES AND FUTURE DEVELOPMENTS}

The DMD array size sets a minimum lateral distance between two uncaging sites for independent gating. For an array size of 15, the minimum distance is $6.5 \mu \mathrm{m}$. This is a soft limit as glutamate uncaging has a much narrower profile $(0.8 \pm 0.1 \mu \mathrm{m}$ lateral FWHM) and considerable overlap between two DMD arrays is needed to induce two-photon uncaging in the OFF site. However, we can further decrease this distance with a slight adjustment in the optical system. By replacing the FT lens (see $\mathrm{L}_{F T}$ in Figure 1A) between the SLM and DMD with one with a shorter focal length, we can achieve smaller foci on the DMD and consequently require smaller DMD array sizes for gating. This, however, will increase the incident power density level on the DMD and will require high-tolerance mirror coatings to prevent damage.

Our system uses a DMD (DLP3000) from an evaluation module (DLP LightCrafter, Texas Instruments). We retained only the components in the module necessary to address the bare DMD via the video interface and removed the other components (e.g., light source and projection lenses). The DMD is electronically driven at $1440 \mathrm{~Hz}$ when delivering the uncaging binary pattern via the computer's digital video output. This rate can be increased up to $4 \mathrm{kHz}(250 \mu \mathrm{s})$ when 96 frames are preloaded onto the onboard memory of the DMD's driver circuit but this would give a total of only $24 \mathrm{~ms}$ of stimulation. Alternatively, one can use more advanced DMD systems (e.g., DLP V-module, ViALUX), which are capable of switching rates of up to $22 \mathrm{kHz}$. One limitation in this work is the low damage threshold of the DMD component itself. Since it is not designed for use with high power light sources (e.g., lasers), illuminating the DMD for long periods with focused laser spots damages the device. Such a technical constraint could be overcome by custom fabricating DMDs with specific mirror coatings to tolerate higher power levels. In this work, the DMD was potentially stretched to its limits by positioning it in the Fourier plane where the holographically projected foci from the $2 \mathrm{P}$ laser were incident. Long sequences of spatio-temporal 
stimulation patterns are not possible with the current device. Nonetheless, even with the limits of the current device it is apparent that the combination of SLM and DMD can be cooperatively applied to the study of synaptic integration.

In summary, holographic projection using an SLM combined with high-speed temporal gating via a DMD allows the generation of random spatio-temporal stimulation patterns in $3 \mathrm{D}$ with submillisecond temporal resolution, and in this way offers unprecedented flexibility in the design of $4 \mathrm{D}$ light patterns for highly localized release of neurotransmitters. Implementing this approach opens up a wide range of prospects for the study of neuronal circuits.

\section{ACKNOWLEDGMENTS}

This work is supported by the Australian Research Council Discovery Project (contract no. DP120102191).

\section{SUPPLEMENTARY MATERIAL}

The Supplementary Material for this article can be found online at: http://www.frontiersin.org/journal/10.3389/fncel.2013. 00231/abstract

Supplementary movie file showing DMD switching of SLMgenerated multi-focal photostimulation pattern.

\section{REFERENCES}

Anselmi, F., Ventalon, C., Begue, A., Ogden, D., and Emiliani, V. (2011). Threedimensional imaging and photostimulation by remote-focusing and holographic light patterning. Proc. Natl. Acad. Sci. U.S.A. 108, 19504-19509. doi: 10.1073/pnas.1109111108

Bednarkiewicz, A., Bouhifd, M., and Whelan, M. P. (2008). Digital micromirror device as a spatial illuminator for fluorescence lifetime and hyperspectral imaging. Appl. Opt. 47, 1193-1199. doi: 10.1364/AO.47.001193

Boyden, E. S., Zhang, F., Bamberg, E., Nagel, G., and Deisseroth, K. (2005). Millisecond-timescale, genetically targeted optical control of neural activity. Nat. Neurosci. 8, 1263-1268. doi: 10.1038/nn1525

Branco, T., Clark, B. A., and Hausser, M. (2010). Dendritic discrimination of temporal input sequences in cortical neurons. Science 329, 1671-1675. doi: 10.1126/science.1189664

Callaway, E. M., and Katz, L. C. (1993). Photostimulation using caged glutamate reveals functional circuitry in living brain slices. Proc. Natl. Acad. Sci. U.S.A. 90, 7661-7665. doi: 10.1073/pnas.90.16.7661

Curtis, J., Koss, B. A., and Grier, D. (2002). Dynamic holographic optical tweezers. Opt. Commun. 207, 169-175. doi: 10.1016/S0030-4018(02)01524-9

Dal Maschio, M., Difato, F., Beltramo, R., Blau, A., Benfenati, F., and Fellin, T. (2010). Simultaneous two-photon imaging and photostimulation with structured light illumination. Opt. Express 18, 18720-18731. doi: 10.1364/OE.18.018720

Daria, V. R., Stricker, C., Bowman, R., Redman, S., and Bachor, H. A. (2009). Arbitrary multisite two-photon excitation in four dimensions. Appl. Phys. Lett. 95, 093791. doi: 10.1063/1.3216581

Dayton, D., Browne, S., Gonglewski, J., and Restaino, S. (2001). Characterization and control of a multi-element dual-frequency liquid-crystal device for highspeed adaptive optical wave-front correction. Appl. Opt. 40, 2345-2355. doi: 10.1364/AO.40.002345

Denk, W. (1994). Two-photon scanning photochemical microscopy: mapping ligand-gated ion channel distributions. Proc. Natl. Acad. Sci. U.S.A. 91, 6629-6633. doi: 10.1073/pnas.91.14.6629

Ducros, M., Houssen, Y. G., Bradley, J., De Sars, V., and Charpak, S. (2013). Encoded multisite two-photon microscopy. Proc. Natl. Acad. Sci. U.S.A. 110, 13138-13143. doi: 10.1073/pnas.1307818110

Fino, E., Araya, R., Peterka, D. S., Salierno, M., Etchenique, R., and Yuste, R. (2009). Rubi-glutamate: two-photon and visible-light photoactivation of neurons and dendritic spines. Front. Neural Circuits 3:2. doi: 10.3389/neuro.04.002.2009
Gasparini, C., and Magee, J. C. (2001). State-dependent dendritic computation in hippocampal cal pyramidal neurons. J. Neurosci. 26, 2088-2100. doi: 10.1523/JNEUROSCI.4428-05.2006

Go, M. A., Ng, P. F., Bachor, H. A., and Daria, V. R. (2011). Optimal complex field holographic projection. Opt. Lett. 36, 3073-3075. doi: 10.1364/OL.36.003073

Go, M. A., Stricker, C., Redman, S., Bachor, H. A., and Daria, V. R. (2012). Simultaneous two-photon imaging and photostimulation with structured light illumination. J. Biophoton. 5, 745-753. doi: 10.1002/jbio.201100101

Katona, G., Szalay, G., Mak, P., Kaszs, A., Veress, M., Hillier, D., et al. (2012). Fast two-photon in vivo imaging with three-dimensional random-access scanning in large tissue volumes. Nat. Methods 9, 201-208. doi: 10.1038/nmeth.1851

Kirby, A., and Love, G. (2004). Fast, large and controllable phase modulation using dual frequency liquid crystals. Opt. Express 12, 1470-1475. doi: 10.1364/OPEX.12.001470

Larkman, A. U. (1991). Dendritic morphology of pyramidal neurons of the rat: spine distributions. J. Comp. Neurol. 306, 332-343. doi: 10.1002/cne.903060209

Liesener, J., Reichester, M., Haist, T., and Tiziani, H. J. (2000). Multi-functional optical tweezers using computer generated holograms. Opt. Commun. 185, 77-82. doi: 10.1016/S0030-4018(00)00990-1

Liu, X., Ramirez, S., Pang, P. T., Puryear, C. B., Govindarajan, A., Deisseroth, K., et al. (2012). Optogenetic stimulation of a hippocampal engram activates fear memory recall. Nature 484, 381-385. doi: 10.1038/nature1 1028

Lovett-Barron, M., Turi, G. F., Kaifosh, P., Lee, P. H., Bolze, F., Sun, X. H., et al. (2012). Regulation of neuronal input transformations by tunable dendritic inhibition. Nat. Neurosci. 15, 423-430. doi: 10.1038/nn.3024

Lutz, C., Otis, T., DeSars, V., Charpak, S., DiGregorio, D., and Emiliani, V. (2008). Holographic photolysis of caged neurotransmitters. Nat. Methods 5, 821-827. doi: 10.1038/nmeth.1241

Matsuzaki, M., Ellis-Davies, G. C. R., Nemoto, T., Miyashita, Y., Iino, M., and Kasai, H. (2001). Dendritic spine geometry is critical for ampa receptor expression in hippocampal cal pyramidal neurons. Nat. Neurosci. 4, 1086-1092. doi: 10.1038/nn736

Megias, M., Emri, Z., Freund, T. F., and Gulyas, A. I. (2001). Total number and distribution of inhibitory and excitatory synapses on hippocampal cal pyramidal cells. Neuroscience 102, 527-540. doi: 10.1016/S0306-4522(00)00496-6

Nagel, G., Szellas, T., Huhn, W., Kateriya, S., Adeishvili, N., Berthold, P., et al. (2003). Channelrhodopsin-2, a directly light-gated cation-selective membrane channel. Proc. Natl. Acad. Sci. U.S.A. 100, 13940-13945. doi: 10.1073/pnas.1936192100

Nevian, T., Larkum, M. E., Polsky, A., and Schiller, J. (2007). Properties of basal dendrites of layer 5 pyramidal neurons: a direct patch-clamp recording study. Nat. Neurosci. 10, 206-214. doi: 10.1038/nn1826

Nikolenko, V., Watson, B., Araya, R., Woodruff, A., Peterka, D., and Yuste, R. (2008). Microscopy: scanless two-photon imaging and photostimulation using spatial light modulators. Front. Neural Circuits 2:5. doi: 10.3389/neuro.04.005.2008

Palima, D., and Daria, V. R. (2006). Effect of spurious diffraction orders in arbitrary multi-foci patterns produced via phase-only holograms. Appl. Opt. 45, 6689-6693. doi: 10.1364/AO.45.006689

Palima, D., and Daria, V. R. (2007). Holographic projection of arbitrary light patterns with a suppressed zero-order beam. Appl. Opt. 46, 4197-4201. doi 10.1364/AO.46.004197

Papagiakoumou, E., Anselmi, F., Bgue, A., de Sars, V., Glückstad, J., Isacoff, E. Y., et al. (2010). Scanless two-photon excitation of channelrhodopsin-2. Nat. Methods 7, 848-854. doi: 10.1038/nmeth.1505

Polsky, A., Mel, B. W., and Schiller, J. (2004). Computational subunits in thin dendrites of pyramidal cells. Nat. Neurosci. 7, 621-627. doi: 10.1038/nn1253

Reddy, G. D., Kelleher, K., Fink, R., and Saggau, P. (2008). Three-dimensional random access multi-photon microscopy for functional imaging of neuronal activity. Nat. Neurosci. 11, 713-720. doi: 10.1038/nn.2116

Reutsky-Gefen, I., Golan, L., Farah, N., Schejter, A., Tsur, L., Brosh, I., et al. (2013). Holographic optogenetic stimulation of patterned neuronal activity for vision restoration. Nat. Commun. 4:1509. doi: 10.1038/ncomms2500

Spruston, N. (2008). Pyramidal neurons: dendritic structure and synaptic integration. Nat. Rev. Neurosci. 9, 206-221. doi: 10.1038/nrn2286

Thalhammer, G., Bowman, R., Love, G., Padgett, M., and Ritsch-Marte, M. (2013). Speeding up liquid crystal slms using overdrive with phase change reduction. Opt. Express 21, 1779-1797. doi: 10.1364/OE.21. 001779 
Wang, S., Szobota, S., Wang, Y., Volgraf, M., Liu, Z., Sun, C., et al. (2007). All optical interface for parallel, remote, and spatiotemporal control of neuronal activity. Nano. Lett. 7, 3859-3863. doi: 10.1021/nl072783t

Wu, S. (1986). Phase retardation dependent optical response time of parallelaligned liquid crystals. J. Appl. Phys. 60, 1836-1838. doi: 10.1063/1.337228

Yang, S., Papagiakoumou, E., Guillon, M., de Sars, V., Tang, C.-M., and Emiliani, V. (2011). Three-dimensional holographic photostimulation of the dendritic arbor. J. Neural Eng. 8:046002. doi: 10.1088/1741-2560/8/4/046002

Zahid, M., Vlez-Fort, M., Papagiakoumou, E., Ventalon, C., Angulo, M. C., and Emiliani, V. (2010). Holographic photolysis for multiple cell stimulation in mouse hippocampal slices. PLoS ONE 5:e9431. doi: 10.1371/journal.pone.0009431

Zhu, P., Fajardo, O., Shum, J., SchŁrer, Y. P. Z., and Friedrich, R. W. (2012). High-resolution optical control of spatiotemporal neuronal activity patterns in zebrafish using a digital micromirror device. Nat. Prot. 7, 1410-1425. doi: $10.1038 /$ nprot.2012.072
Conflict of Interest Statement: The authors declare that the research was conducted in the absence of any commercial or financial relationships that could be construed as a potential conflict of interest.

Received: 20 July 2013; accepted: 06 November 2013; published online: 02 December 2013.

Citation: Go MA, To M-S, Stricker C, Redman S, Bachor H-A, Stuart GJ and Daria VR (2013) Four-dimensional multi-site photolysis of caged neurotransmitters. Front. Cell. Neurosci. 7:231. doi: 10.3389/fncel.2013.00231

This article was submitted to the journal Frontiers in Cellular Neuroscience.

Copyright (C) 2013 Go, To, Stricker, Redman, Bachor, Stuart and Daria. This is an open-access article distributed under the terms of the Creative Commons Attribution License (CC BY). The use, distribution or reproduction in other forums is permitted, provided the original author(s) or licensor are credited and that the original publication in this journal is cited, in accordance with accepted academic practice. No use, distribution or reproduction is permitted which does not comply with these terms. 